\title{
A Lagrangian dispersion model with a stochastic equation for the temperature fluctuations
}

\author{
Andrea Bisignano* \\ Institute of Atmospheric Sciences and Climate (ISAC), \\ National Research Council (CNR), \\ Corso Fiume 4, 10133, Torino, Italy \\ E-mail: a.bisignano@isac.cnr.it

\section{Enrico Ferrero} \\ Dipartimento di Scienze e Innovazione Tecnologica, \\ Università del Piemonte Orientale, \\ Viale Teresa Michel, 11, 15121 Alessandria, Italy \\ E-mail: enrico.ferrero@uniupo.it

\section{Stefano Alessandrini} \\ University Corporation for Atmospheric Research (UCAR), \\ 3090 Center Green Drive, Boulder, CO 80301, USA \\ E-mail: alessand@ucar.edu
}

\begin{abstract}
A new plume rise scheme for the Lagrangian stochastic model SPRAYWEB is developed and tested. The plume rise scheme is based on a stochastic differential equation for the potential temperature fluctuations coupled with the equations for the wind velocity fluctuation components. The new approach is tested against measured data from a water tank experiment (Weil et al., 2002). The results are discussed in term of statistical indices and scatter plots. For the sake of comparison, the new scheme performance is compared with the algorithm used in SPRAYWEB formerly proposed by Anfossi et al. (1993) which doesn't account for the temperature fluctuations. The results obtained with the novel plume rise scheme are generally satisfactory. A better agreement is found for the vertical standard deviation with respect to the results given by the Anfossi et al. (1993) scheme.
\end{abstract}

Keywords: Plume rise, Lagrangian stochastic dispersion model, buoyancy, temperature fluctuations

This paper is a revised and expanded version of a paper entitled [title] presented at the 18th International conference on Harmonisation within Atmospheric Dispersion Modelling for Regulatory Purposes. Bologna, Italy, October, October 9-12, 2017. The paper is submitted for a special issue with Silvana Di Sabatino and Silvia Trini Castelli as guest editors..

Biographical notes: Andrea Bisignano received his Master's in Physics of 


\begin{abstract}
Author
Complex Systems at the University of Torino, Italy and $\mathrm{PhD}$ at the University of Piemonte Orientale during which he spent eight months as visiting researchstudent at the Metoffice, UK. He is now a Temporary Research Fellow at the Institute of Atmospheric Sciences and Climate in Torino, and teaches at the University of the Piemonte Orientale. His main topics of research are particles dispersion models, plume rise and concentration fluctuations.
\end{abstract}

Enrico Ferrero earned his $\mathrm{PhD}$ in Geophysics in 1993, then he became an Assistant Professor at the University of Piemonte Orientale, Italy and since 2006, he is an Associate Professor. He is a Research Collaborator at the Institute for the Sciences of Atmosphere and Climate of the National Research Council (CNR, Italy). His main research fields are: atmospheric physics, planetary boundary layer meteorology, turbulence, atmospheric dispersion modelling, physical and numerical models. He is the author of more than 100 papers published on international journals and conference proceedings.

Stefano Alessandrini is a scientist at the National Center for Atmospheric Research (NCAR), Boulder, Colorado. His main research areas of interest are ind and solar energy probabilistic forecasting, application of air pollution models, environmental physics, turbulence and mesoscale modelling. He has published more than 30 articles regarding probabilistic forecasting of renewable energies and stochastic Lagrangian particle models. He earned his Laurea MS in Physics with Summa Cum Laude from the University of Milano, Italy in 1996 and he received his $\mathrm{PhD}$ at the University of Piemonte Orientale, Italy in 2011 with a topic regarding Lagrangian particle models. Before joining NCAR, he has worked for 12 years in Italy at the Ricerca sul Sistema Energetico (RSE) a public research centre carrying out research activities on mesoscale modelling and probabilistic forecasting of renewable energies.

\title{
1 Introduction
}

Emissions from many natural and anthropogenic sources are hot compared with the surrounding ambient air. These buoyancy effects cause the emitted plume to rise, increasing the effective source height and significantly decreasing the maximum groundlevel concentrations. A significant difference between buoyant and passive dispersion is the buoyant fluid particles ability in creating their own turbulence. Hence, the exchange processes between the plume and its environment need to be accounted for. Models of buoyant plumes originated with the work of Morton et al. (1956). These models describe the mean flow of the plume but do not explicitly take into account for the fluctuations in the velocity and buoyancy that occur within the plume (except through entrainment). In most realistic dispersion models that are used for operational purposes, as the Lagrangian stochastic models (LSMs), the Lagrangian particles move independently of each other through the flow field. There is then an inherent difficulty in modelling a coherent process such as buoyant plume rise using single-particle LSMs: the motion of individual 
Title

particles or fluid elements depends on the buoyancy of all the fluid elements. Moreover, there is nothing to constrain two neighboring model particles to move upwards with similar velocities. Starting from the pioneering work of Briggs (1975), several authors have attempted to model buoyant plume rise using a Lagrangian approach. Three different "hybrid" techniques (i.e., that consider together Lagrangian and Eulerian properties) have been proposed (Webster and Thomson, 2002; Anfossi and Physick, 2005): particles emission occurs at the final plume height computed by analytical models (such as the Briggs 1975 ones), an integral plume rise model is used at each time step for each particle and the derived velocities are added to the Lagrangian stochastic particles velocities (see, for instance, Anfossi et al., 1993) and a set of differential equations describing the time and space evolution of bulk plume quantities are solved at each time step (Webster and Thomson, 2002; Anfossi et al., 2010). An interesting method was proposed by van Dop (1992) in which a Langevin equation for the particle temperature is solved and the buoyancy of the particle is included in the Langevin equation for the particle velocity evolution. Alessandrini et al. 2013 and Ferrero et al. 2017 introduced a fictitious scalar transported by the particles, the temperature difference between the plume portions and the environment air temperature. In this work, the hybrid Lagrangian stochastic algorithm for buoyant plume rise from an isolated source described by Bisignano and Devenish (2015) is introduced into the Lagrangian dispersion model SPRAYWEB (Tinarelli et al, 1994, Alessandrini et al. 2013, Bisignano et al., 2017). In this approach, each particle carries its own potential temperature, which evolves according to a stochastic differential equation similar to Van Dop (1992). The buoyancy is calculated from the particle temperature and is directly included in the equation for the evolution of the velocity through a coupling term. We compare the concentration field simulated by the model with the results of a water tank experiment (Weil et al. 2002) and with a SPRAYWEB simulation carried out with Anfossi et al. (1993) plume rise formulation instead of Bisignano and Devenish (2015) one. In Section 2 the methodology is described, while results are shown and discussed in Section 3. Conclusions are presented in Section 4.

\section{Methodology}

Here we consider a hybrid model introduced by Webster and Thomson (2002) in which the mean flow is calculated from a simple plume model and the fluctuations are calculated using a Lagrangian stochastic model (LSM). Webster and Thomson (2002) only considered fluctuations in the velocity and not the temperature; here we treat both fluctuations of the velocity and temperature as in Bisignano and Devenish (2015). The governing equations for potential temperature and vertical velocity are derived from the Briggs (1984) plume equations. Then we separate the average and the turbulent fluctuating parts of the two variables through the application of the Reynolds decomposition. The final expressions of the stochastic differential equations (SDEs) for 
Author

turbulent vertical velocity and potential temperature of the plume are obtained by adding terms of the form of Thomson (1987) to the turbulent fluctuating parts in order to satisfy the well-mixed condition. Accounting for the fluctuations in temperature means that the Lagrangian particles carry their own potential temperature, which evolves according to an SDE, as in Van Dop (1992). The effect of temperature fluctuations is directly included in the equation for the evolution of the velocity through a coupling term. To our knowledge, an expression for the temporal Lagrangian structure function for a passive scalar is not prescribed in literature. Hence, a completely satisfying approach for setting turbulence parameters for the temperature SDE based on Lagrangian description is not yet available. The constants required in the temperature SDE are set following the values commonly found in literature from both measurements and large eddy simulations (Devenish et al., 2010). The above-described temperature SDE (Bisignano and Devenish, 2015) is introduced for the first time into the LSM SPRAYWEB (Tinarelli et al, 1994, Alessandrini et al. 2013, Bisignano et al., 2017) that, in its standard form, describes the plume rise by using the Anfossi et al. (1993) algorithm. We validate the model against the water tank experiment of Weil et al. (2002) characterized by a strong capping inversion at the top of a convective boundary layer (CBL). We also compared our plume rise approach with that of Anfossi et al. (1993). The focus of Weil et al. (2002) experiment is on highly buoyant plumes that loft near or become trapped in the CBL capping inversion and resist downward mixing. The scaling factors used for calculating the simulation parameters are chosen to be the same as Ferrero et al. (2017).

\subsection{The plume rise model}

The equations governing the rise of a buoyant plume in a uniform crossflow $U[\mathrm{~m} / \mathrm{s}]$ are given by Briggs (1984):

$$
\begin{gathered}
\frac{d}{d s}\left(\pi v b^{2}\right)=E \\
\left(1+k_{v}\right) \frac{d}{d s}\left(\pi v w b^{2}\right)=\pi b^{2} g^{\prime} \\
\frac{d}{d s}\left(\pi v g^{\prime} b^{2}\right)=-N^{2} \pi b^{2} w
\end{gathered}
$$

where $v=\left(U^{2}+w^{2}\right)^{1 / 2}[\mathrm{~m} / \mathrm{s}]$ is the velocity component along the plume axis, $s[\mathrm{~m}]$ is the distance along the plume axis, $E[\mathrm{~m} 2 / \mathrm{s}]$ is the entrainment rate (to be defined), $w$ [m/s] is the vertical velocity of the plume, $b[\mathrm{~m}]$ is the plume radius, $N[1 / \mathrm{s}]$ is the ambient buoyancy frequency and $g^{\prime}=g\left(\theta(\mathrm{z})-\theta_{\mathrm{a}}(\mathrm{z})\right) / \theta_{0}\left[\mathrm{~m} / \mathrm{s}^{2}\right]$ is the reduced gravity in which $\theta(\mathrm{z})$ $[\mathrm{K}]$ is the potential temperature of the plume at height $z[\mathrm{~m}], \theta_{\mathrm{a}}(\mathrm{z})[\mathrm{K}]$ is the ambient potential temperature at height $z$ and $\theta_{0}[\mathrm{k}]$ is a reference temperature. The parameter $k_{\mathrm{v}}$ is the added-mass coefficient that accounts for the momentum of the ambient fluid 
Title

displaced by the plume as the plume rises (here we considered a value of $k_{\mathrm{v}}=1.3$ as suggested by Briggs, 1984). Equations (1) respectively describe the evolution of the volume flux $V\left[\mathrm{~m}^{3} / \mathrm{s}\right]$, momentum flux (per unit density) $M\left[\mathrm{~m}^{4} / \mathrm{s}^{2}\right]$ and the buoyancy flux $F\left[\mathrm{~m}^{4} / \mathrm{s}^{3}\right]$. They are collectively known as the plume equations.

We use the plume equations (1) as a starting for point re-expressing them in terms of $w$, $b, \theta$ and time. First, we expand the left-hand side of equation (1) by applying the product rule for derivatives. Then we express the plume equations in term of $w, b$, and $\theta$, obtaining the expressions for the spatial derivatives of the three quantities. We re-write the derivatives of $w, b$, and $\theta$ with respect to $s$ as derivative with respect to $t=\int d s / v$. Once the time derivatives of $w, b$, and $\theta$, making use of $N^{2}=g / \theta_{0} d \theta_{a} / d z=g /\left(\theta_{0} w\right) d \theta_{a} / d t$ and after some algebra, we get:

$$
\begin{gathered}
\frac{d w}{d t}=\frac{g\left(\theta-\theta_{a}\right)}{\theta_{0}}-E \frac{w}{b^{2}} \\
\frac{d b}{d t}=\frac{E}{2 \pi b}-\frac{b w}{2\left(1+k_{v}\right) v^{2}} \frac{g\left(\theta-\theta_{a}\right)}{\theta_{0}}+\frac{E w^{2}}{2 \pi b v^{2}} \\
\frac{d \theta}{d t}=\frac{-E\left(\theta-\theta_{a}\right)}{b^{2}}
\end{gathered}
$$

The equations (2) reduce to those of a vertically rising plume as $v \rightarrow w$ and to a bent-over plume as $w \rightarrow 0$. These equations are now used to calculate the mean velocity and temperature (which will be denoted by an overbar). The fluctuating velocity and temperature are denoted by a prime and will be calculated from SDEs. These are constructed from analogous equations to (2) and coupled with LSMs for $w^{\prime}$ and $\theta^{\prime}$. Let us show the application of a Reynolds decomposition to the equations for $w$ and $\theta$. Because equations (2) are linear in $w$ and $\theta$ there are no second-order quantities and there is no feedback on the mean quantities by the fluctuating quantities. Let us assume, first, that $b^{\prime}=0$ and hence that is evaluated in terms of the mean quantities alone and, second, that $E=E(w ; v ; U)$. Since we assume also that there are no fluctuations in $\theta_{\mathrm{a}}$, the Reynolds decompositions for $w$ and $\theta$ are:

$$
\begin{gathered}
\frac{d}{d t}\left(w^{\prime}+\bar{w}\right)=g \frac{\left(\bar{\theta}-\theta_{a}\right)}{\theta_{0}}+g \frac{\theta^{\prime}}{\theta_{0}}-E \frac{\bar{w}}{b^{2}}-E \frac{w^{\prime}}{b^{2}} \\
\frac{d}{d t}\left(\bar{\theta}+\theta^{\prime}\right)=-E \frac{\left(\bar{\theta}-\theta_{a}\right)}{b^{2}}-E \frac{\theta^{\prime}}{b^{2}}
\end{gathered}
$$

Then the SDEs for $w^{\prime}[\mathrm{m} / \mathrm{s}]$ and $\theta^{\prime}[\mathrm{K}]$ are completed by adding respectively the LSM of Thomson (1987) for inhomogeneous turbulence and an LSM (in which for simplicity we assume that the temperature statistics are homogeneous) similar to that considered by van Dop (1992): 


$$
\begin{gathered}
d w^{\prime}=g \frac{\theta^{\prime}}{\theta_{0}} d t-E \frac{w^{\prime}}{b^{2}} d t-\frac{w^{\prime}}{T_{L}} d t+\frac{1}{2}\left(\frac{1}{w}+\frac{w^{\prime}}{\sigma_{w}^{2}}\right) d \sigma_{w}^{2}+\sqrt{C_{0} \epsilon} d W \\
d \theta^{\prime}=-E \frac{\theta^{\prime}}{b^{2}} d t-\frac{\theta^{\prime}}{T_{\theta}} d t-\frac{w^{\prime}}{w} d \bar{\theta}+\sqrt{C_{\theta} \epsilon_{\theta}} d W_{\theta}
\end{gathered}
$$

where $T_{\mathrm{L}}[\mathrm{s}]$ and $T_{\theta}[\mathrm{s}]$ are the time scales on which $w^{\prime}$ and $\theta^{\prime}$ decorrelates respectively, $\varepsilon$ $\left[\mathrm{m}^{2} / \mathrm{s}^{2}\right]$ and $\varepsilon_{\theta}\left[\mathrm{K}^{2}\right]$ are the mean kinetic energy and scalar dissipation rates respectively, $C_{\theta}=1.6$ is the Obukhov-Corrsin constant, $\sigma_{\mathrm{w}}[\mathrm{m} / \mathrm{s}]$ is the vertical-velocity standard deviation and $C_{0}$ is the constant of proportionality in the second-order Lagrangian velocity structure function which typically has a value in the range 5-7 in homogeneous isotropic turbulence. This range for $C_{0}$ is commonly found experimentally and from direct numerical simulations of homogeneous isotropic turbulence. We choose $C_{0}=5$ because it gives the best agreement with the data. The value is smaller than $C_{0}=6$ used in Bisignano and Devenish (2015). The small difference can be due to the different Reynolds-number flows that characterizethe two case studies. The third term of RHS of equation (4) for $\theta$ ' arises from the time derivative of mean temperature that implicitly contains fluctuations of velocity. This term and the first term of RHS together ensure that $\theta$ is conserved following a particle, in both equations (4) the term involving $\mathrm{E}$ and the fluctuating quantities represent the effect of the entrainment on the turbulence, whereas the terms involving the time scales represent the internal turbulence of the plume. We neglect the covariance $\sigma_{w \theta}[\mathrm{m} / \mathrm{s} \mathrm{K}]$ that may exist in reality.

The definition of entrainment, as in Bisignano and Devensih (2015), includes two additive entrainment mechanisms in a crosswind, one due to velocity differences parallel to the plume axis and the other due to velocity differences normal to the plume axis and that the two mechanisms are additive. Devenish et al. (2010) suggested that this additive entrainment assumption be an $l^{m}$-norm.

$$
E=2 \pi b\left[\alpha\left(|w| \frac{|w|}{v}\right)^{m}+\beta\left(|w| \frac{U}{v}\right)^{m}\right]^{1 / m}
$$

We have assumed that the difference between the horizontal component of the plume velocity and $U$ is small relative to $U$ and that this is valid from the source. Note that entrainment is proportional to the absolute velocity difference in order to avoid spurious detrainment after the plume reaches its maximum rise height (Bisgnano and Devenish 2015). The constant coefficients $\alpha$ and $\beta$ are associated with the two entrainment mechanisms: $\alpha$ with velocity differences parallel to the plume axis and $\beta$ with velocity differences normal to the plume axis. We take $\alpha=0.1$ and $\beta=0.5$ which are consistent with previous studies (Devenish et al., 2010). $m>1$ is a tunable parameter. The effect of a crossflow on a buoyant plume can be characterized by the dimensionless parameter $\tilde{U}=$ $U /\left(F_{b} N\right)^{1 / 4}$ where $F_{b}$ is the source buoyancy flux. In the weak-wind limit, $\tilde{U}<<1$, the first 
term on the right-hand side of equation (5) dominates. When $\tilde{U}>>1$, the plume becomes bent-over and the second term on the right-hand side of equation (5) dominates. Devenish et al. (2010) found that $m=3 / 2$ gave the best agreement with the LES of buoyant plumes in a crosswind and field observations. We use this value throughout this study. The turbulence parameters $\sigma_{\mathrm{w}}, \sigma_{\theta}, T_{\mathrm{L}}$ and $T_{\theta}$ have been chosen to be related to the appropriate mean quantities in the problem. Also, it is necessary to limit the turbulence parameters in order to avoid numerical overflow in the oscillating region. We set:

$$
\begin{gathered}
\sigma_{w}=\alpha \max \left(|\bar{w}|, \bar{w}^{*}\right) \\
T_{L}=\frac{b}{\max \left(|\bar{w}|, \bar{w}^{*}\right)} \\
\sigma_{\theta}=\gamma \max \left(\left|\bar{\theta}-\theta_{a}\right|, \bar{\theta}^{*}\right) \\
T_{\theta}=T_{L} \\
\text { with } \\
\bar{w}^{*}=2^{-5 / 8} \pi^{-1 / 4}(6 / 5 \alpha)^{-1}(9 / 10 \alpha)^{1 / 2} F_{0}^{1 / 4} N^{1 / 4} \\
\bar{\theta}^{*}=2^{-5 / 4} \pi^{-1 / 4}(5 / 8 \alpha)^{1 / 2} \theta_{0} g^{-1} F_{0}^{1 / 4} N^{5 / 4} \\
b^{*}=\left(2^{3 / 4}-2^{3 / 8}\right) \pi^{-1 / 4}(9 / 10 \alpha)^{-1 / 2} F_{0}^{1 / 4} N^{-3 / 4}
\end{gathered}
$$

where $\alpha$ and $\gamma$ are tunable constants whose values are chosen equal to 0.1 , and $F_{0}$ the initial buoyancy flux. The initialization of $w, b$ and $g^{\prime}$ (denoted with a subscript 0 ) for a pure plume whose initial buoyancy flux is known is not straightforward. We estimate $w_{0}$ by equating the initial radius $b_{0}=2 z$ so that $w_{0}=\left(b_{0} g_{0}^{\prime}\right)^{1 / 2}$. Since the initial buoyancy flux is $F_{0}=\pi \mathrm{b}^{2}{ }_{0} g_{0}^{\prime} v_{0}$ we obtain a cubic polynomial for either $w_{0}$ or $g_{0}^{\prime}$ for given $b_{0}$, whose roots can be inferred by analyzing the discriminant $\Delta$. Any of the three cases $(\Delta>0, \Delta=0, \Delta<0)$ will produce a physical solution. In the case that there are three real roots $(\Delta>0)$, shows that two of these roots will be negative, and can thus be discarded (Bisignano and Devenish (2015) for further details).

\subsection{The case study}

We considered the Weil et al. (2002) experiment which investigates the plume dispersion in the convective boundary layer (CBL) using a convection tank. The focus is on highly buoyant plumes that become trapped in the CBL capping inversion and resist downward mixing. Such plumes are defined by a dimensionless buoyancy flux $F_{*}>0.1 . F_{*}$ is defined as:

$$
F_{*}=\frac{F_{b}}{U w_{*}^{2} Z_{i}} \quad \text { with } \quad F_{b}=w_{s} r_{s}^{2} g \frac{\rho_{s}-\rho_{a}}{\rho_{a}}=w_{s} r_{s}^{2} g \frac{T_{a}-T_{s}}{T_{s}}
$$


Author

where $F_{\mathrm{b}}$ is the stack buoyancy flux, $U$ is the mean wind speed, $\mathrm{w}_{*}$ is the convective velocity scale, $z_{\mathrm{i}}$ is the CBL depth, $\rho$ the density, $T$ the temperature, $r$ is the source radius, $w$ the plume velocity at the source, and $g$ the gravity acceleration (" $s$ " stands for stack values and " $a$ " for ambient air values). In particular, the experiment is characterized by four different values of the normalized stack buoyancy flux $\left(F_{*}=0, F_{*}=0.1, F_{*}=0.2\right.$, and $\left.F_{*}=0.4\right)$. We tested the plume-rise models of Anfossi (1993) and of Bisignano and Devenish (2015) against the observations as a function of $F_{*}$ and of the dimensionless downwind distance

$$
X=\frac{x U}{w_{*} Z_{i}}
$$

We underline that in the experiment there was no mean flow and, hence, no environment mechanical turbulence. This condition may lead to an underestimation of the entrainment in the experiment when compared to both the real atmosphere and our simulations. Our turbulence parametrizations assume the presence of mechanical turbulence. Consequently, a slight underestimation of the plume rise may occur in our simulations, when compared to that observed in the water tank. Ferrero et al. (2017) estimated the absence of the mechanical turbulence to influence the observed final plume rise by much less than 20\%. The experiment reproduces allthe components of the lateral and vertical dispersion parameters (rms meander, relative dispersion, total dispersion), mean and rootmean-square concentration fields as a function of $\mathrm{F}_{*}$ for continuous buoyant releases.

We perform our simulations only for the cases $F_{*}=0.1$ and $F_{*}=0.2$ (hereinafter named case A and case B). In fact, we are not interested in non-buoyant emission $\left(F_{*}=0\right)$ and, for the case $F_{*}=0.4$, the experimental data for vertical profiles of dimensionless crosswind-integrated concentration are not available. To perform the simulations, we rescaled the experiments to typical atmospheric dimensions as in Ferrero et al. (2017). The main experimental parameters are listed in the first column of Table 1 and the rescaled simulation parameters in the second column. In Table $1 N=0.5 \mathrm{~s}^{-1}$ is the BruntVaisala frequency of the inversion layer above the mixing height, $N_{*}=N z_{i} / W_{*}$ its dimensionless value, $F_{m}=w_{s}^{2} r_{s}^{2} \rho_{s} / \rho_{a}$ the stack momentum flux, $F_{m^{*}}=F_{m} /\left(u z_{i}^{2} w_{*}\right)$ its dimensionless value, $F_{b^{*}}$ and $\mathrm{F}_{b}$ correspond to eq. 9 and $g^{\prime}=g \Delta$ is the reduced gravity and

$\Delta$ is the relative density. The turbulence parameters have been determined following the same scheme as Ferrero et al. (2017) and they are listed in Table 2. Here, $\sigma_{\mathrm{u}}(z), \sigma_{\mathrm{v}}(z)$ and $\sigma_{w}(z)$ are the three components of the wind standard deviation; $T_{L u}(z)=T_{L v}(z)=T_{L w}(z)$ are the Lagrangian time scale along the three directions; $\left\langle w^{3}\right\rangle$ is third order moment of the vertical velocity perturbations. The dimensions of variables involved in this section are reported in Table 1 and Table 2. 


\section{Results}

We focus on the plume characteristics and on the vertical profiles of dimensionless crosswind-integrated concentration as a function of dimensionless downwind distance for two $F_{*}$ values $\left(F_{*}=0.1\right.$, case A and $F_{*}=0.2$, case B). We compare the measured and the simulated results, both with Anfossi et al. (1993) plume rise and Bisignano and Devenish (2015) plume rise. Figures 1-2 (that refers to the cases A and B respectively) show that the model plume characteristics agree well with the data plume characteristics for both the values of normalized buoyancy flux. Thus, the model is able to correctly reproduce the basic behavior of the plume rise phenomenon in convective conditions. In particular, we considered the following plume dispersion parameters: mean height, horizontal and vertical standard deviations; all are normalized by the inversion height $z_{i}$ and plotted as a function of dimensionless distance $X$. The model shows a little overestimation both for the vertical and lateral standard deviation and an underestimation in the mean height. We found that the vertical spread, evaluated with the above-described plume rise, matches the data slightly better than that evaluated with the Anfossi et al. (1993) plume rise, characterized by the absence of temperature fluctuations. There is no significant difference between the two plume rise algorithms for lateral standard deviation, as both the method affect only the vertical component of the motion of the particles. Regarding the mean height, on the contrary, the better agreement with the data results has been obtained by Anfossi et al. (1993) plume rise algorithm. Similar considerations can be drawn looking at Figures 3-4, where scatter diagrams report the same data, but where the simulations results are evaluated at the same dimensionless distance of the measurements. Again, the scatter plots show that the overall agreement between measure and simulation results is good for both the values of normalized buoyancy flux and all the points are within the factor two curves (except for a pint in the mean height scatter). Also Figures 34 further confirm that both the models underestimate the mean height, overestimate the standard deviations and the Bisignano and Devenish (2015) plume rise better agrees with the data that the Anfossi et al. (1993) one.

Figures 5-6 (that refers to the cases A and B respectively) show the comparison between the measured (Weil et al. 2002) and simulated (both with Anfossi et al., 1993 and Bisignano and Devenish, 2015 plume rise methods) vertical profiles of dimensionless crosswind-integrated concentration as a function of dimensionless downwind distance. The non-dimensionalization of concentration has been made by use of the experimental parameters (Weil et al. 2002) and it takes the form $C_{y} U z_{i} / Q$, where $Q$ is the source strength and $C_{y}$ is the crosswind-integrated concentration, i.e.:

$$
C_{y}=\int C(x, y, z) d y
$$

The agreement is satisfactory at all dimensionless downwind distance. We considered the profiles within a normalized distance $X=2$ as the ambient-plume temperature difference is not significant at larger distances from the source acting as a source of buoyancy. This allows also to avoid possible numerical overflows with the Bisignano and Devenish 
Author

(2015) plume rise method, which is very sensitive to ambient-plume temperature difference. In the $F_{*}=0.1$ case, the agreement between the height of the peaks of concentration evaluated by the models and those measured by Weil et all. (2002) deteriorate as the downwind distance increases. This discrepancies for increasing distances are also notable near the ground, where the two models underestimate the data concentration profiles. The last statement seems to hold true for Figure 6 as well. Conversely, with the stronger buoyancy flux $\left(F_{*}=0.2\right)$, we observe a better agreement between the simulated (with both the models) and measured peaks locations, at all dimensionless downwind distance. Furthermore, in this case, comparison of Bisignano and Devenish (2015) method with Anfossi et al. (1993) formulation, i.e. with no temperature fluctuations, shows that the first one had a greater vertical spread than the first one results. This is much more noticeable than in Case $\mathrm{A}$, as the temperature fluctuations evaluated by Bisignano and Devenish (2015) algorithm increase as the buoyancy flux become larger. For sake of comparison, in Figures 7-8 we show the scatter diagrams of the measurements against the simulations for dimensionless crosswindintegrated concentration, for the case $\mathrm{A}$ and $\mathrm{B}$ respectively. Here, all the considered downwind distances ( $X=0.5, X=1, X=1.5$, and $X=2)$ are plotted together. The scatterplots lead to the same conclusions that we made for Figures 5-6. We notice that there is a noticeable number of points outside the factor two curves. Most of them (in the low part of the diagrams) represent a model underestimation of the data (for both the two models). These points refer to the lower part of the vertical profiles where both the models are not able to capture the values of data concentrations. A small number of points outside the two-factor curves are located in the upper part of the scatter diagrams for which, therefore, the models overestimate the data. These points refer to the upper levels of the profiles where the overestimation is due to the above-mentioned shift of the peak locations. In Table 3, the statistical analysis is presented for crosswind-integrated concentration. In particular, the correlation (Corr), the normalized mean squared error (NMSE) and fractional bias (FB) are evaluated. For both the models, all the three indexes are more satisfactory for the higher value of buoyancy flux (Corr increases, NMSE and the absolute value of FB decrease). We detect that the absolute value of FB (which is the only index that estimates only systematic errors which lead to always underestimate or overestimate the measured values) is lower for Anfossi et al. (2003) in the Case A, on the contrary it is lower for Bisignano and Devenish (2015) in Case B.

\section{Conclusions}

The hybrid model of buoyant plume rise combines coupled SDEs for vertical velocity and temperature with a classical plume model of a buoyant rise in a crossflow. The novelty lies in adding a stochastic differential equation (SDE) and a $w^{\prime}-\theta^{\prime}$ coupling term to the SPRAYWEB model. With these equations, the model can account for the turbulence generated by the plume itself. The model can reproduce the basic behavior of the plume rise phenomenon in convective conditions. In fact, there is a good agreement with Weil et al. (2002, Boundary-Layer Meteorol.) water tank experiment in terms of the 
Title

dispersion parameters. In particular, the model shows a little overestimation for the vertical standard deviation, a little underestimation of the mean height and of the vertical concentration profiles near the ground. Some discrepancies were observed in the heights of the peak of the concentration for the low-buoyancy case, but the agreement improves for the high-buoyancy case. The comparison of the Bisignano and Devenish (2015) with the Anfossi et al. (1993) formulation shows that the former produces a larger vertical spread of the particles than the latter. The simulations with Bisignano and Devenish (2015) plume rise algorithm lead to a better agreement with the vertical standard deviation data than those carried out with of Anfossi et al. (1993) formulation which doesn't account for the temperature fluctuations.

\section{References}

Alessandrini S., Ferrero F., Anfossi D., 2013, A new Lagrangian method for modelling the buoyant plume rise, Atmos. Environ., 77, 239-249

Anfossi, D., Ferrero, E., Brusasca, G., Marzorati, A., Tinarelli, G., 1993. A simple way of computing buoyant plume rise in a lagrangian stochastic model for airborne dispersion. Atmos. Environ. 27A (9), 1443-1451.

Anfossi, D., Physick, W., 2005. Lagrangian particle models. In: Zannetti, P. (Ed.), Chapter 11 of Air Quality Modeling Theories, Methodologies, Computational Techniques, and Available Databases and Software. Fundamentals, vol. II. The EnviroComp Institute and the Air \& Waste Management Association, pp. 93-161.

Anfossi, D., Tinarelli, G., Trini Castelli, S., Nibart, M., Olry, C., Commanay, J., 2010. A new Lagrangian particle model for the simulation of dense gas dispersion. Atmos. Environ. 44, 753-762

Bisignano, A., Devenish, B., 2015, A model for temperature fluctuations in a buoyant plume. Boundary-Layer Meteorology, 57, 2, p157

Bisignano, A., Ferrero, E., Alessandrini S., Mortarini, L., 2017. Int. J. Environ. Pollut. Model chain for buoyant plume dispersion, Int. J. Environ. Pollut., 62, 2/3/4, 200-213.

Briggs, G.A. 1984. Plume rise and buoyancy effects. In: Randerson D (ed), Atmospheric science and power production, Office of Research, US Department of Energy, Washington, 327366

Devenish, B.J., Rooney, G.G., Thomson, D.J. 2010. Large-eddy simulation of a buoyant plume in uniform and stably stratified environments. J Fluid Mech. 652, 75-103

Ferrero, E., Alessandrini S., Anfossi D., 2017. Lagrangian simulations of the plume rise in strong capping inversion. Int. J. Environ. Pollut., 62, 2/3/4, 184-198

Morton, B.R., G.I. Taylor, and J.S. Turner (1956): Turbulent gravitational convection from maintained and instantaneous sources. Proc. Roy. Soc. London, A234, 1-23.

Tinarelli G., Anfossi D., Brusasca G., Ferrero E., Giostra U., Morselli M.G., Moussafir J., Tampieri F., Trombetti F., 1994. Lagrangian particle simulation of tracer dispersion in the lee of a schematic two-dimensional hill, Journal of Applied Meteorology, 33, 744-756 


\section{Author}

Thomson, D.J. 1987. Criteria for the selection of stochastic models of particle trajectories in turbulent flows. J Fluid Mech 180, 529-556

van Dop, H., 1992. Buoyant plume rise in a Lagrangian Framework. Atmos. Environ, 26A 1335-346

Webster, H.N., Thomson, D.J. 2002. Validation of a Lagrangian model plume rise scheme using the Kincaid data set. Atmos Environ 36, 5031-5042

Weil C. J., Snyder W. H., Lawson R. E. JR. and Shipman M. S. (2002) Experiments on buoyant plume dispersion in a laboratory convection tank, Bound.-Layer Meteor., 102, 367-414. 
Title

\begin{tabular}{|c|c|c|}
\hline & Experiments & Simulations \\
\hline \multicolumn{3}{|l|}{ CASE A } \\
\hline$z i$ & $200 \mathrm{~mm}$ & $1000 \mathrm{~m}$ \\
\hline$r_{s}$ & $1.59 \mathrm{~mm}$ & $7.95 \mathrm{~m}$ \\
\hline$z_{s}$ & $30 \mathrm{~mm}$ & $150 \mathrm{~m}$ \\
\hline$w_{s}$ & $99.4 \mathrm{~mm} \mathrm{~s}^{-1}$ & $19.88 \mathrm{~m} \mathrm{~s}^{-1}$ \\
\hline$w *$ & $7.4 \mathrm{~mm} \mathrm{~s}^{-1}$ & $1.48 \mathrm{~m} \mathrm{~s}^{-1}$ \\
\hline$u$ & $20.7 \mathrm{~mm} \mathrm{~s}^{-1}$ & $4.14 \mathrm{~m} \mathrm{~s}^{-1}$ \\
\hline$N$ & $0.5 \mathrm{~s}^{-1}$ & $0.002 \mathrm{~s}^{-1}$ \\
\hline$\partial \theta_{a} / \partial z$ aloft & $0.007725 \mathrm{~K} \mathrm{~mm}^{-1}$ & $1.5510^{-3} \mathrm{~K} \mathrm{~m}^{-1}$ \\
\hline$F_{m}$ & $25121.52 \mathrm{~mm}^{4} \mathrm{~s}^{-2}$ & $25121.52 \mathrm{~m}^{4} \mathrm{~s}^{-2}$ \\
\hline$F_{m} *$ & $4.110^{-3}$ & $4.110^{-3}$ \\
\hline$N^{*}$ & 13.51 & 13.51 \\
\hline$F_{b^{*}}$ & 0.1 & 0.1 \\
\hline$F_{b}$ & $22670.64 \mathrm{~mm}^{4} \mathrm{~s}^{-3}$ & $906.8 \mathrm{~m}^{4} \mathrm{~s}^{-3}$ \\
\hline$g^{\prime}$ & $90.22 \mathrm{~mm} \mathrm{~s}^{-2}$ & $0.72 \mathrm{~m} \mathrm{~s}^{-2}$ \\
\hline$\Delta$ & $9.2010^{-3}$ & $9.2010^{-3}$ \\
\hline$\rho_{s}$ & $0.986 \mathrm{Kg} \mathrm{m}^{-3}$ & $0.922 \mathrm{Kg} \mathrm{m}^{-3}$ \\
\hline$\rho_{s} / \rho_{a}$ & 0.9908 & 0.9264 \\
\hline$T_{s}$ & $306.0 \mathrm{~K}$ & $327.2 \mathrm{~K}$ \\
\hline \multicolumn{3}{|l|}{ CASE B } \\
\hline$z i$ & $200 \mathrm{~mm}$ & $1000 \mathrm{~m}$ \\
\hline$r_{s}$ & $1.59 \mathrm{~mm}$ & $7.95 \mathrm{~m}$ \\
\hline$Z_{s}$ & $30 \mathrm{~mm}$ & $150 \mathrm{~m}$ \\
\hline$w_{s}$ & $99.4 \mathrm{~mm} \mathrm{~s}^{-1}$ & $19.88 \mathrm{~m} \mathrm{~s}^{-1}$ \\
\hline$W_{*}$ & $7.4 \mathrm{~mm} \mathrm{~s}^{-1}$ & $1.48 \mathrm{~m} \mathrm{~s}^{-1}$ \\
\hline$u$ & $20.7 \mathrm{~mm} \mathrm{~s}^{-1}$ & $4.14 \mathrm{~m} \mathrm{~s}^{-1}$ \\
\hline$N$ & $0.5 \mathrm{~s}^{-1}$ & $0.002 \mathrm{~s}^{-1}$ \\
\hline$\partial \theta_{a} / \partial z$ aloft & $0.007725 \mathrm{~K} \mathrm{~mm}^{-1}$ & $1.5510^{-3} \mathrm{~K} \mathrm{~m}^{-1}$ \\
\hline$F_{m}$ & $25121.52 \mathrm{~mm}^{4} \mathrm{~s}^{-2}$ & $25121.52 \quad \mathrm{~m}^{4} \mathrm{~s}^{-2}$ \\
\hline$F_{m}{ }^{*}$ & $4.110^{-3}$ & $4.110^{-3}$ \\
\hline$N^{*}$ & 13.51 & 13.51 \\
\hline$F_{b^{*}}$ & 0.2 & 0.2 \\
\hline$F_{b}$ & $45341.29 \mathrm{~mm}^{4} \mathrm{~s}^{-3}$ & $1813.651 \mathrm{~m}^{4} \mathrm{~s}^{-3}$ \\
\hline$g^{\prime}$ & $180.4319 \mathrm{~mm} \mathrm{~s}^{-2}$ & $1.443455 \mathrm{~m} \mathrm{~s}^{-2}$ \\
\hline$\Delta$ & $1.8410^{-2}$ & $1.8410^{-2}$ \\
\hline$\rho_{s}$ & $0.977 \mathrm{Kg} \mathrm{m}^{-3}$ & $0.849 \mathrm{Kg} \mathrm{m}^{-3}$ \\
\hline$\rho_{s} / \rho_{a}$ & 0.982 & 0.853 \\
\hline$T_{s}$ & $308.8 \mathrm{~K}$ & $355.5 \mathrm{~K}$ \\
\hline
\end{tabular}

Table 1: Values of the parameters for the experiments and simulations 
Author

\begin{tabular}{|l|l|}
\hline Height & $0<\mathrm{z}<\mathrm{z}_{\mathrm{i}}(0<\mathrm{z}<800 \mathrm{~m})$ \\
\hline \multicolumn{2}{|l|}{ Note: as suggested by Weil et al. (2002) } \\
\hline$\sigma_{\mathrm{u}}=\sigma_{\mathrm{v}}$ & $0.6 \mathrm{~W}_{*}=1.48 \cdot 0.6=0.888 \mathrm{~ms}^{-1}$ \\
\hline$\sigma_{\mathrm{w}}$ & $0.6 \mathrm{~W}_{*}=1.48 \cdot 0.6=0.888 \mathrm{~ms}^{-1}$ \\
\hline $\mathrm{T}_{\mathrm{Lu}}(\mathrm{z})=\mathrm{T}_{\mathrm{Lv}}(\mathrm{z})=\mathrm{T}_{\mathrm{Lw}}(\mathrm{z})$ & $0.6 \mathrm{z}_{\mathrm{i}} \mathrm{W}_{*}=405.4 \mathrm{~s}$ \\
\hline$<\mathrm{W}^{3}>$ & $0.8 \sigma_{\mathrm{w}}{ }^{3} \mathrm{~m}^{3} \mathrm{~s}^{-3}$ \\
\hline Height & $1.2 \mathrm{z}_{\mathrm{i}}<\mathrm{z}<1.71 \mathrm{z}_{\mathrm{i}}(1200 \mathrm{~m}<\mathrm{z}<1710 \mathrm{~m})$ \\
\hline Note : all the parameters were set at their minimum $^{2}$ & $0.1 \mathrm{~ms}^{-1}$ \\
\hline$\sigma_{\mathrm{u}}=\sigma_{\mathrm{v}}$ & $0.05 \mathrm{~ms}^{-1}$ \\
\hline$\sigma_{\mathrm{w}}$ & $4000 \mathrm{~s}$ \\
\hline $\mathrm{T}_{\mathrm{Lu}}(\mathrm{z})=\mathrm{T}_{\mathrm{Lv}}(\mathrm{z})=\mathrm{T}_{\mathrm{Lw}}(\mathrm{z})$ & $0.8 \sigma_{\mathrm{w}}{ }^{3} \mathrm{~m}^{3} \mathrm{~s}^{-3}$ \\
\hline$<\mathrm{W}^{3}>$ & $0.8 \mathrm{z}_{\mathrm{i}}<\mathrm{z}<1.2 \mathrm{z}_{\mathrm{i}}(800 \mathrm{~m}<\mathrm{z}<1200 \mathrm{~m})$ \\
\hline Height & \\
\hline $\begin{array}{l}\text { Note: all the parameters were linearly interpolated between the value at } \mathrm{z}=0.8 \mathrm{z}_{\mathrm{i}} \\
\text { and their minimum values. }\end{array}$ & \\
\hline
\end{tabular}

Table 2: Turbulence parameters 

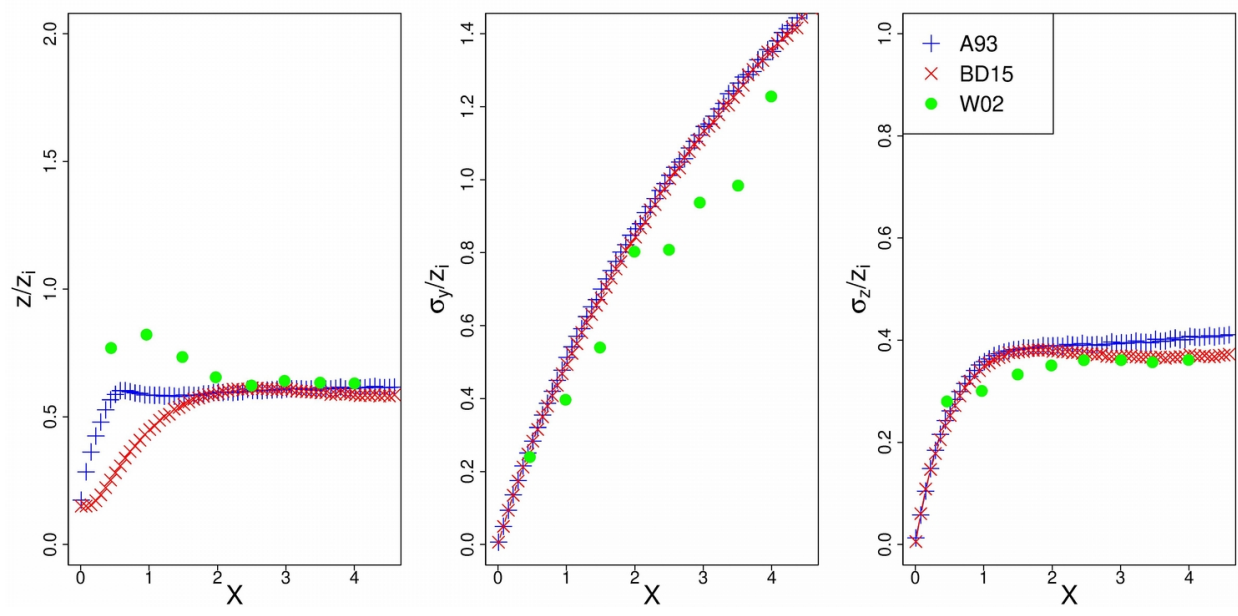

Figure 1. Comparison of the measured (Weil et al. 2002 - W02 in the legend) and simulated (both with plume rise methods of Anfossi et al., 1993 - A93 in the legend and Bisignano and Devenish, 2015 - BD15 in the legend) mean height, horizontal and vertical standard deviations for $F_{*}=0.1$.
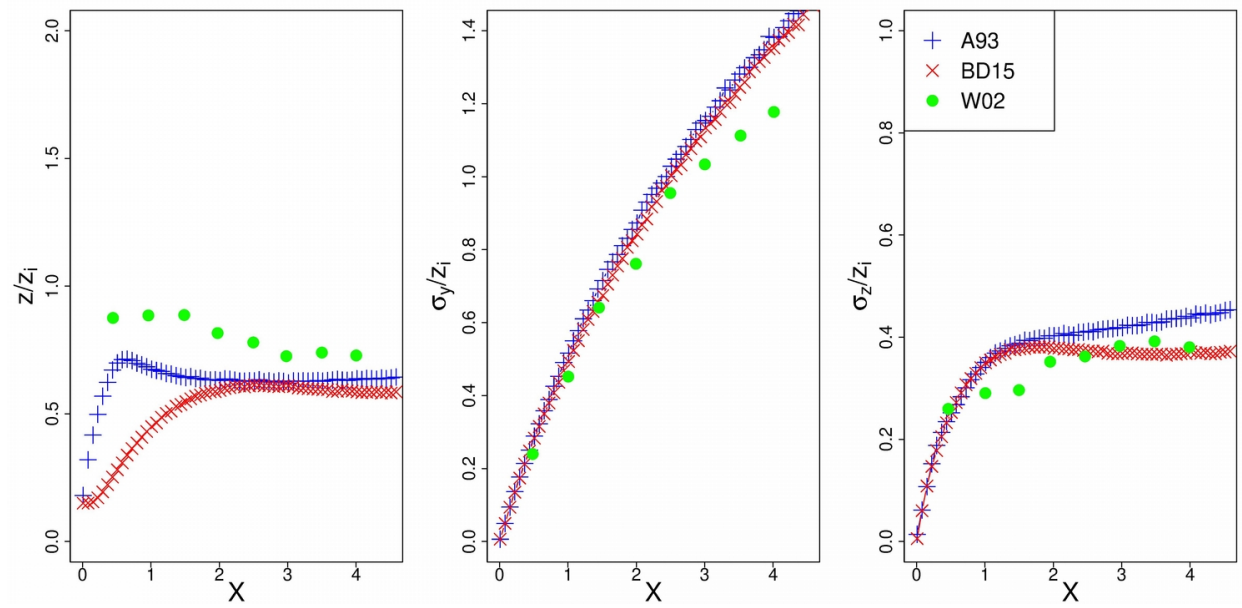

Figure 2. As in Figure 1, but for $F_{*}=0.2$ 


\section{Author}
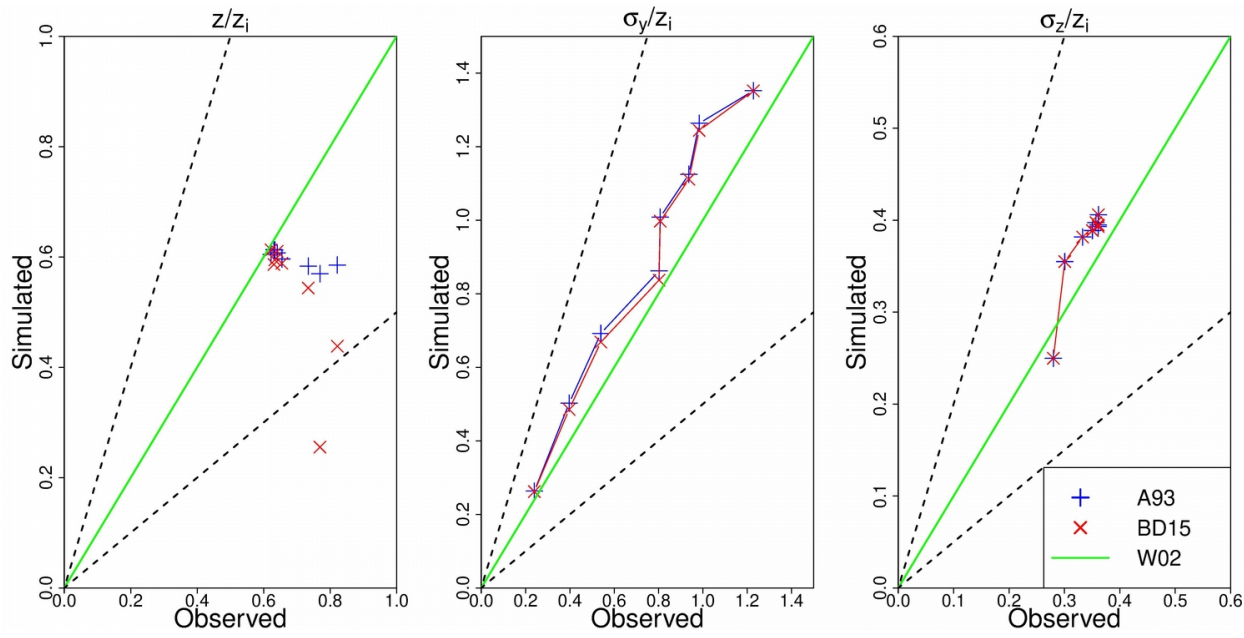

Figure 3. Scatterplots of the measurements (Weil et al. 2002 - W02 in the legend) against the simulations (both with plume rise methods of Anfossi et al., 1993 - A93 in the legend and Bisignano and Devenish, 2015 - BD15 in the legend) for mean height, horizontal and vertical standard deviations in the case $F_{*}=0.1$. The green line represents the perfect agreement, while the black dashed lines are the factor 2 range.
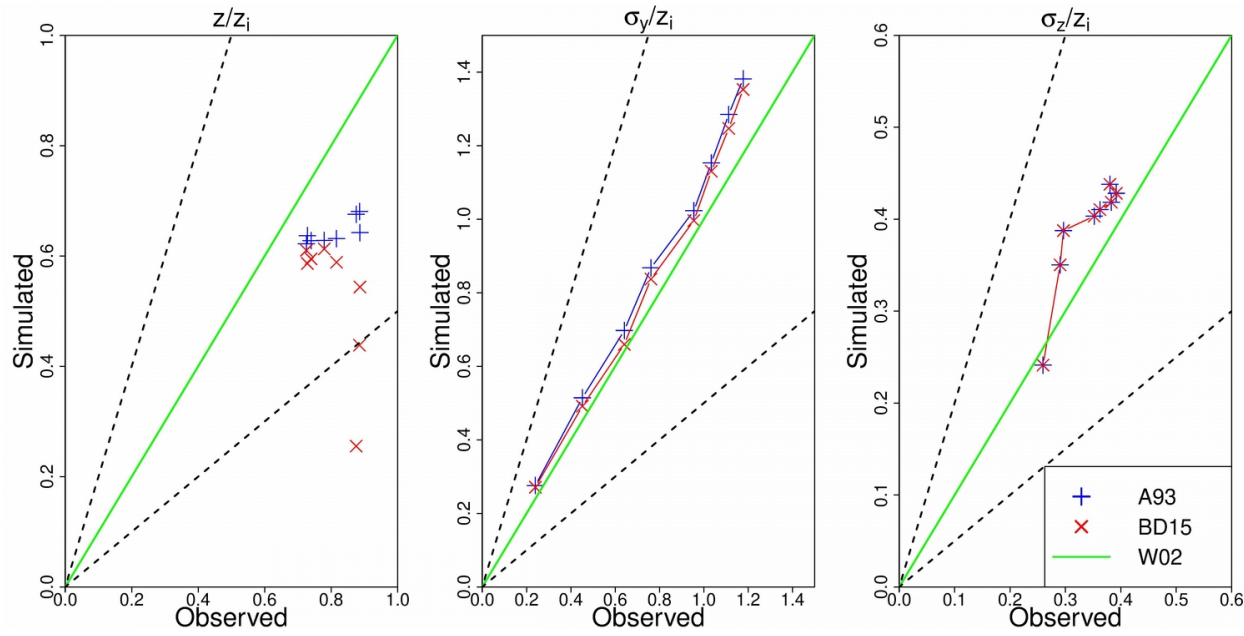

Figure 4. As in Figure 3, but for $F_{*}=0.2$ 
Title
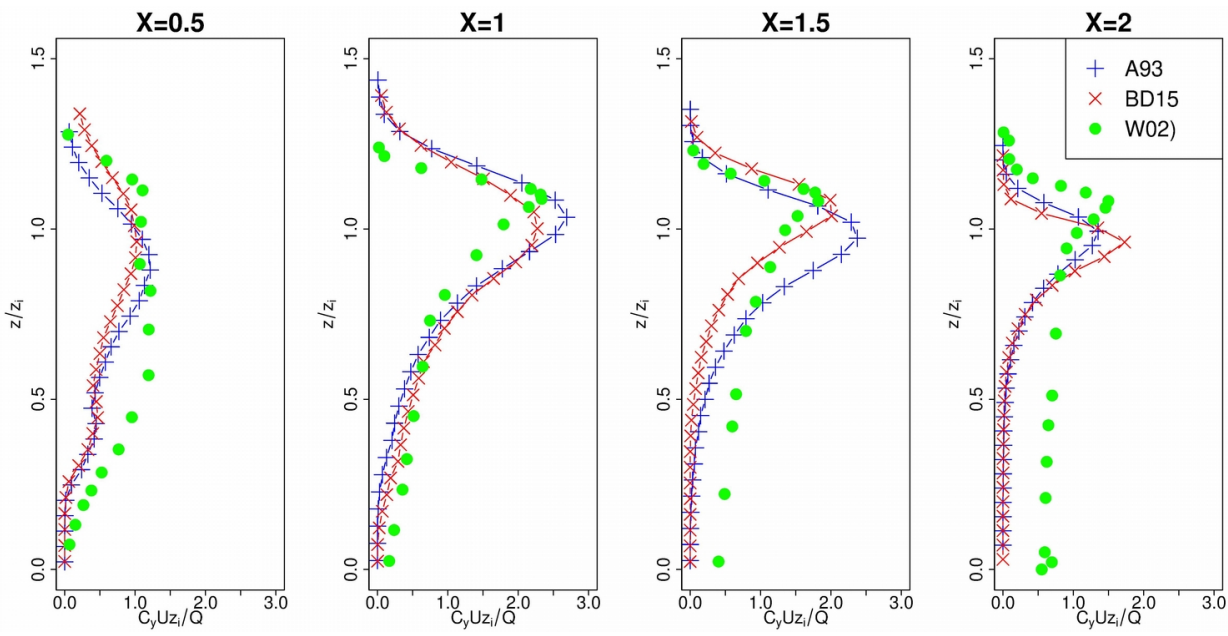

Figure 5. Comparison of the measured (Weil et al. 2002 - W02 in the legend) and simulated (both with plume rise methods of Anfossi et al., 1993 - A93 in the legend and Bisignano and Devenish, 2015 - BD15 in the legend) vertical profiles of dimensionless crosswind-integrated concentration as a function of the dimensionless downwind distance for $F_{*}=0.1$.
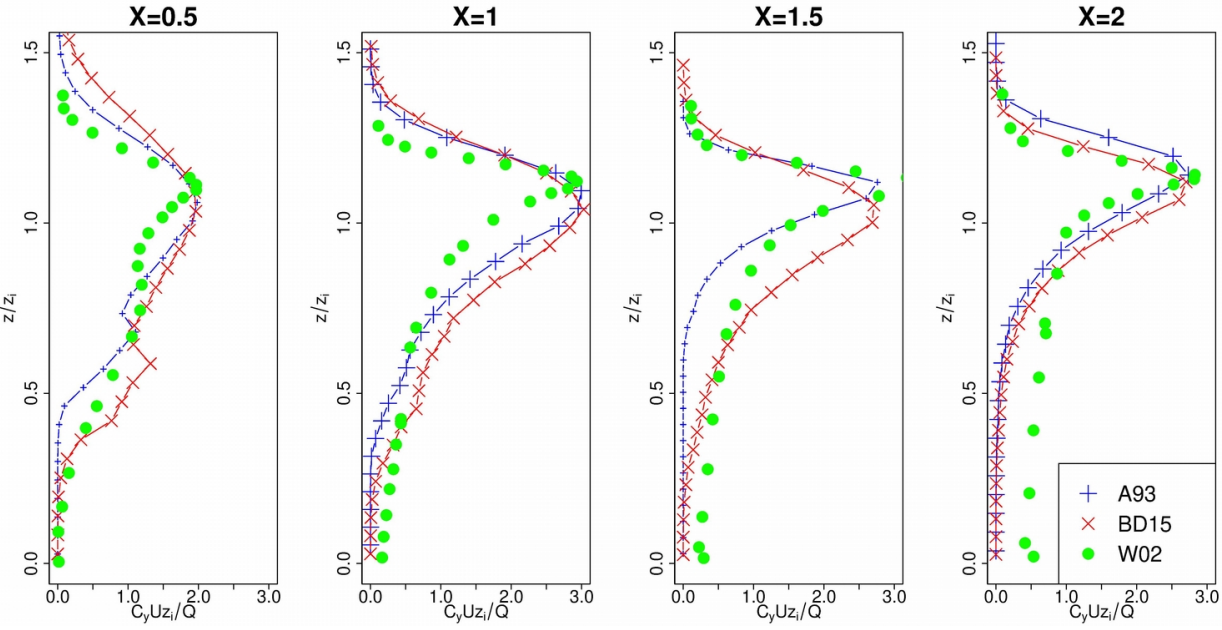

Figure 6. As in Figure 5, but for $F_{*}=0.2$ 
Author

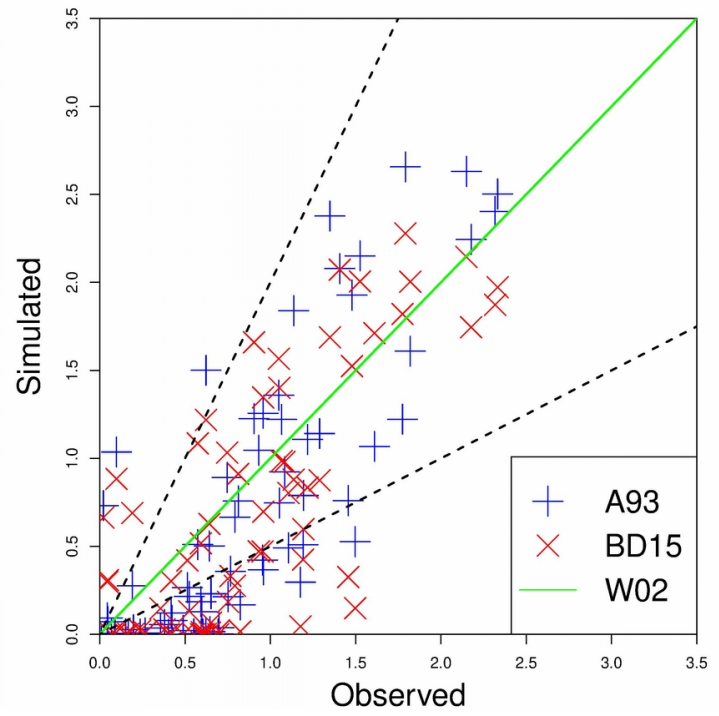

Figure 7. Scatterplots of the measurements (Weil et al. 2002 - W02 in the legend) against the simulations (both with plume rise methods of Anfossi et al., 1993 - A93 in the legend and Bisignano and Devenish, 2015 - BD15 in the legend) for dimensionless crosswind-integrated concentration in the case $F_{*}=0.1$. The green line represents the perfect agreement, while the black dashed lines are the factor 2 range.

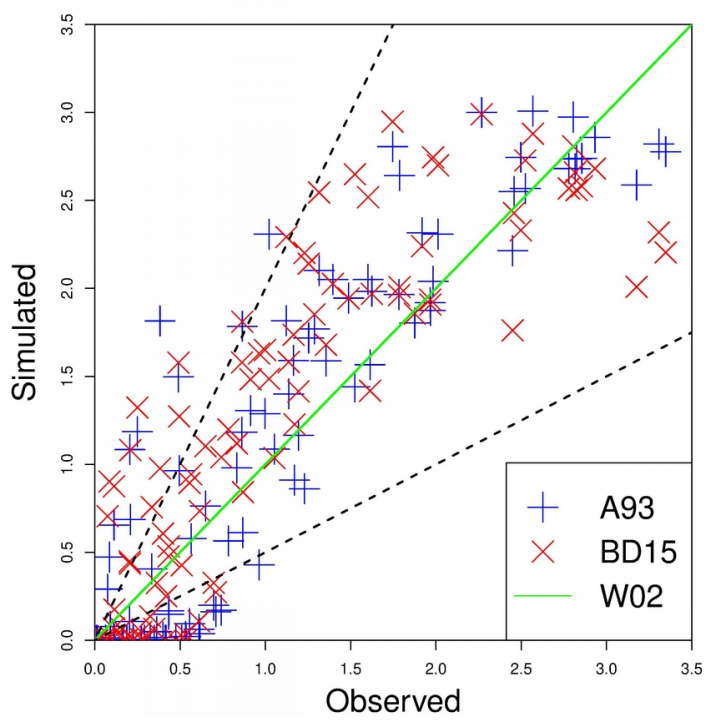

Figure 8. As in Figure 7, but for $F_{*}=0.2$ 


\begin{tabular}{|c|c|c|c|c|c|}
\hline Plume rise method & Quantity & $\mathrm{F}_{*}$ & Corr & NMSE & FB \\
\hline A93 & $\mathrm{C}_{\mathrm{y}} \mathrm{Uz}_{\mathrm{i}} / \mathrm{Q}$ & 0.1 & 0.81 & 0.23 & 0.18 \\
\hline BD15 & $\mathrm{C}_{\mathrm{y}} \mathrm{Uz}_{\mathrm{i}} / \mathrm{Q}$ & 0.1 & 0.76 & 0.27 & 0.23 \\
\hline A93 & $\mathrm{C}_{\mathrm{y}} \mathrm{Uz}_{\mathrm{i}} / \mathrm{Q}$ & 0.2 & 0.90 & 0.10 & -0.048 \\
\hline BD15 & $\mathrm{C}_{\mathrm{y}} \mathrm{Uz}_{\mathrm{i}} / \mathrm{Q}$ & 0.2 & 0.85 & 0.13 & -0.015 \\
\hline
\end{tabular}

Table 3: Statistical indexes for the non-dimensional crosswind integrated concentration 